\title{
La publicidad en buscadores de las plataformas españolas de compraventa de trabajos académicos: análisis del tráfico, costes y palabras clave
}

\author{
Rubén Comas-Forgas*, Mercè Morey-López*, Jaume Sureda-Negre* \\ * Universidad de las Islas Baleares, España \\ Correo-e: rubencomas@uib.es | ORCID iD: https://orcid.org/0000-0002-8885-753X \\ Correo-e: merce.morey@uib.es | ORCID iD: https://orcid.org/0000-0002-2050-6602 \\ Correo-e: sureda.negre@gmail.com | ORCID iD: https://orcid.org/0000-0003-2689-5626
}

Recibido: 31-03-20; 2a versión: 24-06-20; Aceptado: 09-07-20; Publicado: 07-06-21

Cómo citar este artículo/Citation: Comas-Forgas, R.; Morey-López, M., Sureda-Negre, J. (2021). La publicidad en buscadores de las plataformas españolas de compraventa de trabajos académicos: análisis del tráfico, costes y palabras clave. Revista Española de Documentación Científica, 44 (3), e298. https://doi.org/ 10.3989/redc.2021.3.1767

Resumen: Los portales de compraventa de trabajos académicos se han convertido en una suerte de colaboradores necesarios en las prácticas deshonestas del alumnado universitario a nivel mundial y en los últimos años su presencia se ha incrementado notablemente. Nuestro estudio, basado en los datos extraídos del programa SEMrush, analiza el tráfico, la publicidad online y las palabras clave empleadas por 36 empresas españolas dedicadas a la venta de trabajos académicos a través de Internet. Los resultados obtenidos ponen de manifiesto el incremento de visitas que reciben estos sitios web durante el último año, a la vez que ponen el acento en la mayor tasa de tráfico recibido a través de la publicidad online frente al tráfico orgánico. Nuestro aporte concluye con una serie de recomendaciones generales que entendemos pueden ayudar a encarar el problema.

Palabras clave: motores de búsqueda; publicidad online; Google Ads; deshonestidad académica; publicidad en buscadores; educación superior.

\section{Advertising in search engines of the Spanish contract cheating websites: analysis of traf- fic, costs and keywords}

Abstract: The contract cheating websites have become a sort of necessary collaborators in the dishonest practices carried out by university students worldwide and in recent years its presence has increased significantly. Our study, based on data extracted from SEMrush, analyses traffic, online advertising and the marketing keywords used by 36 Spanish companies dedicated to the sale of academic essays and dissertations through the Internet. The results obtained show the increase in visits received by these websites in the last year, while emphasizing the higher rate of traffic received through online advertising compared to organic traffic. Our contribution concludes with a series of general recommendations that we understand can help addressing the problem.

Keywords: search engines; online advertisements; Google Ads; academic dishonesty; search engines advertising; higher education.

Copyright: (c) 2021 CSIC. Este es un artículo de acceso abierto distribuido bajo los términos de la licencia de uso y distribución Creative Commons Reconocimiento 4.0 Internacional (CC BY 4.0). 


\section{INTRODUCCIÓN Y ANTECEDENTES}

La adquisición de trabajos académicos por parte del alumnado a cambio de una remuneración económica constituye una conducta de deshonestidad académica (Comas, 2009) que en la última década ha sido objeto de creciente interés, controversia y debate en el ámbito de la Educación Superior (Newton, 2018). Para referirse a esta práctica se han utilizado diversos conceptos, siendo los más habituales los de "servicios de escritura académica", "escritura fantasma", "compraventa de trabajos académicos", "suplantación en los procesos de evaluación" o "fábricas de trabajos académicos". En esencia, los servicios de compraventa de trabajos académicos ofrecen la realización de tareas personalizadas de casi cualquier disciplina, aunque de entre la variada oferta que presentan los trabajos escritos son el producto más común (Draper y otros, 2017). El alumnado/cliente, en muchos de los servicios existentes, puede especificar la calificación que desea (aunque no haya garantía de cumplimiento de esta cláusula), solicitar borradores, estilos y normativas académicas de referencia y casi cualquier otra característica personalizada que se requiera (Newton y Lang, 2016). Los servicios disponibles son rápidos y asequibles (Wallace y Newton, 2014) y los estudiantes que los utilizan habitualmente subestiman o desconocen la severidad con la que las universidades penalizan este tipo de prácticas fraudulentas (Newton, 2018).

En la actualidad conviven cuatro grandes modalidades de "contratación" de servicios de escritura académica. La primera, que pervive como reminiscencia de la época anterior al uso extensivo de las Tecnologías de la Información y Comunicación (TIC), se realiza a través de los contactos personales con los que un alumno ofrece o demanda a otro un trabajo académico a cambio de una suma de dinero u otro tipo de compensación. La segunda modalidad consiste en acceder a los llamados "redactores fantasma" a través de páginas o portales de anuncios por palabras (también llamados Digital Market Places).

Basta acceder a portales como "Mil Anuncios" y buscar "TFG" para comprobar la abundante oferta existente. La tercera modalidad se desarrolla a través de empresas dedicadas a la redacción de trabajos académicos; compañías a menudo dadas de alta en el registro mercantil y que cuentan con plataformas digitales. La cuarta alternativa consiste en escritores fantasma independientes que suelen hacer uso de los servicios de subastas en línea para anunciar y publicitar su trabajo (Sivasubramaniam y otros, 2016).

Así, lo que no hace muchas décadas era un fraude artesanal en el que sólo existía la modalidad dominada por los contactos personales, se ha transformado en una práctica de dimensiones cuasi-industriales, mediatizada por potentes portales de Internet detrás de los cuales hay empresas o particulares. En este contexto la compraventa de trabajos académicos se ha convertido en un gravísimo problema a nivel mundial que afecta a la eficacia y reputación de las instituciones de enseñanza superior (Foltýnek y Králíková, 2018). Este artículo se centra en la tercera de las modalidades de acceso a los servicios de escritura académica: las plataformas de empresas de redacción de trabajos académicos, y más específicamente a aquellas registradas en España.

Cabe destacar que la literatura sobre el tema se ha orientado, básicamente, en tres direcciones. La primera se ha centrado en el estudio de la prevalencia e incidencia del fenómeno (Bretag y otros, 2020; Curtis y Clare, 2017) y las causas asociadas a su aparición y expansión, tanto desde la óptica del alumnado (Devlin y Gray, 2007; Gullifer, 2010) como desde la perspectiva del profesorado (Awdry y Newton, 2019). La segunda orientación empírica se ha focalizado en la búsqueda y evaluación de estrategias y dispositivos de actuación para combatir este fenómeno (Clare y otros, 2017; Dawson y Sutherland-Smith, 2018, 2019; Draper y Newton, 2017), mientras que la tercera se ha dirigido a aportar conocimiento sobre las características, naturaleza y modo de obrar del mercado de compraventa de trabajos o actividades académicas (Amigud, 2020; Ellis y otros, 2018; Rowland y otros, 2018). Nuestro estudio se circunscribe a esta tercera línea de investigación, centrándose en el impacto de la publicidad de los portales de compraventa de trabajos académicos. Se trata de una dimensión del tema escasamente estudiada y cuyo análisis puede contribuir a justificar tomas de decisiones que pongan coto al mercado de servicios de escritura fantasma en España.

En este artículo se analiza el impacto de los anuncios online en las visitas a los portales españoles de escritura académica. El mercado a través de Internet se ha convertido en una plataforma comercial de primer orden con lo que la publicidad en línea ha pasado a ser la forma más eficiente para dar a conocer los sitios web y promocionar sus productos o servicios. La publicidad en línea se canaliza, básicamente, a través de anuncios de motores de búsqueda (conocida como SEA, acrónimo de "search engines advertising") y anuncios de visualización también llamada "publicidad display" (Kireyev y otros, 2016).

Con los primeros se consigue posicionar información sobre el negocio o producto en la primera página de los resultados de búsqueda, mientras que con los segundos se envían mensajes publicitarios cuando el usuario accede a videos, cuando consulta un periódico online, cuando revisa el correo elec- 
trónico, etc. Las empresas pequeñas y medianas suelen preferir los anuncios de los motores de búsqueda porque están más personalizados; inciden en personas que, por la consulta a los buscadores, ya han mostrado un interés por el producto. Por otro lado, los anuncios de visualización, gráficos o de display son más útiles para grandes compañías, con presupuestos más amplios y que desean retener su impacto entre los clientes y que pueden permitirse no afinar tanto en la búsqueda del grupo objetivo de sus mensajes (Goldfarb y Tucker, 2011). Hay varias plataformas de publicidad online controladas por grandes compañías de motores de búsqueda: Google, Microsoft, Yahoo!, entre otras. En este artículo nos centramos en la plataforma de publicidad Ads de Google, sin duda la más conocida y la que cuenta con una mayor cuota de mercado. En 2019, Google promedió una participación neta global del $73,8 \%$ para búsquedas de escritorio y más del $90 \%$ de la participación neta en búsquedas de dispositivos móviles, lo que la convierte en la plataforma líder de marketing de búsqueda de pago en el mundo (Chaffey, 2020). En un contexto global, Google obtuvo el $78 \%$ de los ingresos de búsqueda de pago en 2017, posicionándose como una de las industrias más grandes y rentables del mundo (Marvin, 2017).

El empleo de técnicas de búsqueda de pago o estrategias de precio o pago por clic (PPC) son fenómenos relativamente nuevos en el marketing. A mediados de la década de 1990, las "Páginas Amarillas" de los EEUU vendieron su inventario publicitario a varias páginas de resultados de buscadores (Search Engine Result Pages, SERP), lo que permitió a los primeros SERP como Overture la capacidad de vender posiciones de búsqueda a empresas (Jansen y Spink, 2007). Estos SERP surgieron inicialmente como las fuentes más útiles para encontrar información en Internet, pero al poco tiempo se convirtieron en plataformas cada vez más valiosas para la publicidad de pago, servicio que les aporta ingentes beneficios (Dreller, 2010).

A medida que los métodos de búsqueda de pago aumentaron en popularidad a mediados de la década de 2000, se desarrollaron cuatro técnicas o estrategias de publicidad en los principales SERP (Sen, 2005). En un primer momento, surgieron los anuncios publicitarios relacionados con palabras clave; las empresas compraban un anuncio en una determinada página de resultados de búsqueda, dependiendo de las palabras clave utilizadas por el usuario de Internet. En un segundo momento, las empresas pagaron por enviar su sitio web a los principales SERP para asegurarse de que su portal se mostrara en las páginas de resultados de búsqueda. Posteriormente, en una tercera fase, las empresas practicaron la optimización de motores de búsqueda (SEO), evaluando búsquedas comunes de Internet por hora, ubicación y palabra clave y modificando sus sitios web para hacerlos más relevantes para búsquedas particulares por parte de sus potenciales consumidores. Finalmente, las empresas pagaron por la ubicación de los resultados de búsqueda, la forma más rápida de obtener visibilidad del sitio web. Por ejemplo, una determinada empresa puede comprar un término (palabra clave) o una cadena (conjunto de palabras clave ordenadas) para garantizar que cuando un usuario ingrese ese término o cadena en un SERP, el usuario vea esa empresa más cerca de la parte superior de la búsqueda resultados.

En las últimas dos décadas, la optimización de motores de búsqueda ha venido siendo una estrategia común para que las empresas dirijan el tráfico de usuarios hacia su sitio web (Baye y otros, 2016; Zhang y Cabage, 2017). Como resultado, esta estrategia ha producido competencia entre empresas similares (Baye y otros, 2016) que han trabajado para optimizar sus sitios web. Se ha descubierto que la optimización de motores de búsqueda es más rentable que los PPC en mercados escasamente competitivos debido a las limitaciones naturales de los consumidores que buscan bienes y servicios particulares (Aswani y otros, 2018; Kritzinger y Weideman, 2017). Sin embargo, a medida que aumenta la competencia en un mercado, las estrategias de búsqueda de pago aumentan su rendimiento (Nabout y otros, 2014; Jafarzadeh y otros, 2019), con una competencia cada vez mayor que hace prácticamente imposible que los anunciantes con presupuestos limitados mantengan las primeras posiciones en las páginas de resultados de búsqueda (Boughton, 2005; Moran y Hunt, 2014), generándose así una suerte de "efecto Mateo".

\section{OBJETIVOS}

Este trabajo se centra en el estudio de diversos aspectos de la publicidad en los motores de búsqueda que insertan las plataformas españolas de escritura académica. La hipótesis que orienta el trabajo señala, en primer lugar, que en los últimos años estas plataformas han conocido un aumento de tráfico y que en este incremento los anuncios han tenido y tienen una alta incidencia. En segundo lugar, sugiere que las palabras clave utilizadas por los anuncios de estos portales constituyen una incitación al fraude académico. En tercer lugar, apunta que estos portales invierten una considerable cantidad de recursos económicos a la publicidad en buscadores.

De acuerdo con la hipótesis señalada, se plantean dos objetivos:

1.- Identificar y analizar el tráfico generado hacia los portales españoles de compraventa 
de trabajos académicos, distinguiendo el que se realiza a partir de búsquedas orgánicas del que se hace a través de anuncios insertados en buscadores (concretamente en Google Ads).

2.- Identificar y analizar las palabras clave o cadenas utilizadas en estos anuncios.

Para la consecución de los dos objetivos señalados se da respuesta a las siguientes preguntas de investigación:

- ¿Cuál es el volumen de anuncios de las plataformas españolas dedicadas a la compraventa de trabajos académicos insertados en Google? ¿Qué diferencias temporales por meses se observan en la inserción de estos anuncios?

- ¿Cuál es el tráfico promedio (anual y mensual) que reciben los portales de compraventa de trabajos académicos en España? ¿Desde qué países se realiza este tráfico? ¿Existen diferencias entre el volumen de tráfico a través de escritorio y de dispositivos móviles?

- ¿Cuál es el volumen de tráfico generado por búsquedas orgánicas? ¿Cuál es el volumen de tráfico que tiene su origen en los anuncios?

- ¿Cuál es el tiempo medio de las visitas a los portales de escritura académica y cuál es su tasa de rebote?

- ¿Cuál es el coste de la publicidad de los portales españoles de compraventa de trabajos académicos?

- ¿Qué palabras clave de pago usan los portales para publicitar sus servicios de escritura académica? ¿Qué volumen de tráfico se genera con estas palabras? ¿Qué coste tiene y a cuántas urls dirigen?

\section{METODOLOGÍA}

\subsection{Método, material y procedimiento}

Los datos se han obtenido a través de SEMrush, una plataforma de gestión de visibilidad online. Este programa utiliza datos de Google Analytics y permite examinar, entre otros aspectos, estrategias de publicidad pagada, agrupación y gestión de palabras clave, efectividad de precio por clic y optimización de motores de búsqueda de sitios web. La utilización de SEMrush ha demostrado su utilidad en trabajos empíricos de características similares al que se presenta en este artículo (Drivas y otros, 2017; Gavilán y otros, 2018; González-Soltero y otros, 2015; Taylor, 2020).

Dado que uno de los objetivos de este trabajo consiste en conocer la incidencia de la publicidad en el tráfico que reciben los portales de escritura fantasma españoles, la primera acción consistió en identificar aquellos sitios web que se anunciaron en Google Ads a lo largo de los últimos 12 meses (entre Marzo de 2019 y Febrero de 2020). Para ello se empleó el análisis de palabras clave utilizándose los descriptores "Trabajo de Fin de Grado", "TFG", "Trabajo de Fin de Máster", "TFM" y "Tesis". Así, y accediendo a todos los anuncios insertados con estas tres palabras o acrónimos, se identificaron 38 portales que los utilizaban. Para el análisis se eliminaron 2 al comprobar que no estaban estrictamente dedicados a la compraventa de trabajos sino a labores de asesoría y formación.

Una vez identificados los 36 elementos de la muestra objeto de estudio se procedió a su análisis siguiendo el siguiente procedimiento:

- A partir de la opción "Análisis del dominio" de SEMrush se obtuvieron datos relativos, en primer lugar, al tráfico orgánico o "natural"; es decir: al número de visitantes procedentes de los resultados de búsqueda. En segundo lugar, los relativos al tráfico de pago del dominio; esto es: el número de visitantes procedentes de anuncios de Google Ads. También se obtuvieron datos sobre el tráfico de escritorio (visitas recibidas desde dispositivos no móviles) el tráfico móvil ${ }^{1}$ (visitantes desde dispositivos móviles) y el coste de la publicidad mensual de cada portal.

- A partir de la opción "Análisis del tráfico" se obtuvieron datos relativos a la duración media de visita a los portales para un periodo de seis meses (de Septiembre de 2019 a Febrero de 2020). También se analizó la tasa de rebote (métrica que arroja el porcentaje de visitantes que abandonan una web cuando entran) así como el tráfico por países.

Por otra parte, se procedió al examen de las palabras clave de los anuncios; es decir, al análisis de aquellas consultas textuales "enviadas por los usuarios a los motores de búsqueda con la finalidad de obtener páginas web relevantes a su consulta" (Aregay, 2014). Para ello (y utilizando la herramienta de SEMrush "Investigación de la publicidad") se extrajeron las palabras clave usadas en los anuncios de los 36 portales objeto de estudio que hacían referencia explícita a la compraventa de trabajos (por ejemplo: "comprar TFG", "cuánto cuesta un TFG", "TFM precio", etc.), desechándose aquellas que no se referían abiertamente a estos servicios (por ejemplo: "TFG de derecho", "TFM", "ejemplo de TFG", etc.). Así se obtuvieron un total de 39 palabras clave o cadenas de palabras que componen la muestra de la segunda parte del estudio. De cada palabra clave se analizó: el PPC; el promedio mensual de veces que los internautas habían buscado cada palabra y, finalmente, el número de direccio- 
nes URL mostradas en los resultados de búsquedas orgánicas por cada palabra clave.

El trabajo de campo fue llevado por los tres investigadores firmantes del artículo (dos investigadores se repartieron el análisis de los portales) y el tercer investigador se centró en extraer los datos relativos a la parte del estudio referido a las palabras clave. El trabajo de campo se desarrolló a lo largo del mes de Marzo de 2020.

\subsection{Análisis y procesamiento de datos}

Todos los datos obtenidos se fusionaron en una matriz SPSS para su análisis estadístico. Estos datos fueron: a) tráfico generado en los portales de compraventa de trabajos académicos; b) duración media y tasa de rebote de las visitas a los portales entre Agosto de 2019 y Febrero de 2020; c) procedencia geográfica del tráfico total; d) palabras clave usadas en los anuncios de Ads (PPC de las palabras clave compradas por los portales en Google Ads, volumen de búsqueda que generan las palabras clave contratadas). De todos estos datos se realizó un análisis estadístico de carácter descriptivo (frecuencias, porcentajes y medias); aparte se usó la prueba estadística Chi-cuadrado de Pearson para calcular la relación existente entre el tráfico de pago recibido por los portales analizados en función del número de anuncios publicados por éstos. Para determinar la relación entre el número de anuncios y el tráfico de pago recibido se llevó a cabo una codificación de los datos generados por SEMrush a partir de dos variables. Una variable independiente (número de anuncios) con tres posiciones concordantes con los márgenes explicitados en el comentario de la Tabla
II, y una variable dependiente (tráfico de pago recibido) que se calculó sumando el tráfico de pago de los portales y ordenándolos en 3 niveles: a) portales con tráfico igual o superior a $5.500(n=11)$; b) los que presentan un tráfico entre 1.200 y 5.499 $(n=12) y$, finalmente, c) portales con tráfico de búsqueda de pago inferior a $1.200(n=13)$.

\section{RESULTADOS}

\subsection{Anuncios insertados en Google Ads por los portales españoles de compraventa de trabajos académicos}

La Tabla I recoge datos referidos a los portales que durante los 12 meses previos al estudio insertaron anuncios en Google Ads (un total de 761 anuncios). Tal y como puede comprobarse, existen grandes diferencias en cuanto al número de anuncios contratados por los diferentes portales, moviéndose en una horquilla que va de los 58 de la plataforma gabinetedeestudios.com a solo uno de otras cuatro. Así, y en relación al posicionamiento de los portales por el número de anuncios, pueden establecerse tres grupos de portales: a) con alta incidencia publicitaria, aquellos que publicaron más de 40 anuncios (del 1 al 10); b) con incidencia media, portales que insertaron entre 10 y 39 anuncios (del 11 al 20); y c) con incidencia baja, portales con menos de 10 anuncios (del 21 al 36).

En cuanto al tipo de producto o servicio que se ofrece en los anuncios, se observa que los más publicitados son los TFG, con 516 anuncios con esta palabra clave, muy por encima de la publicidad centrada en servicios de escritura de TFM (238

Figura 1: Tráfico recibido por los portales de compraventa de trabajos académicos a partir de búsquedas de escritorio y búsquedas de móviles.

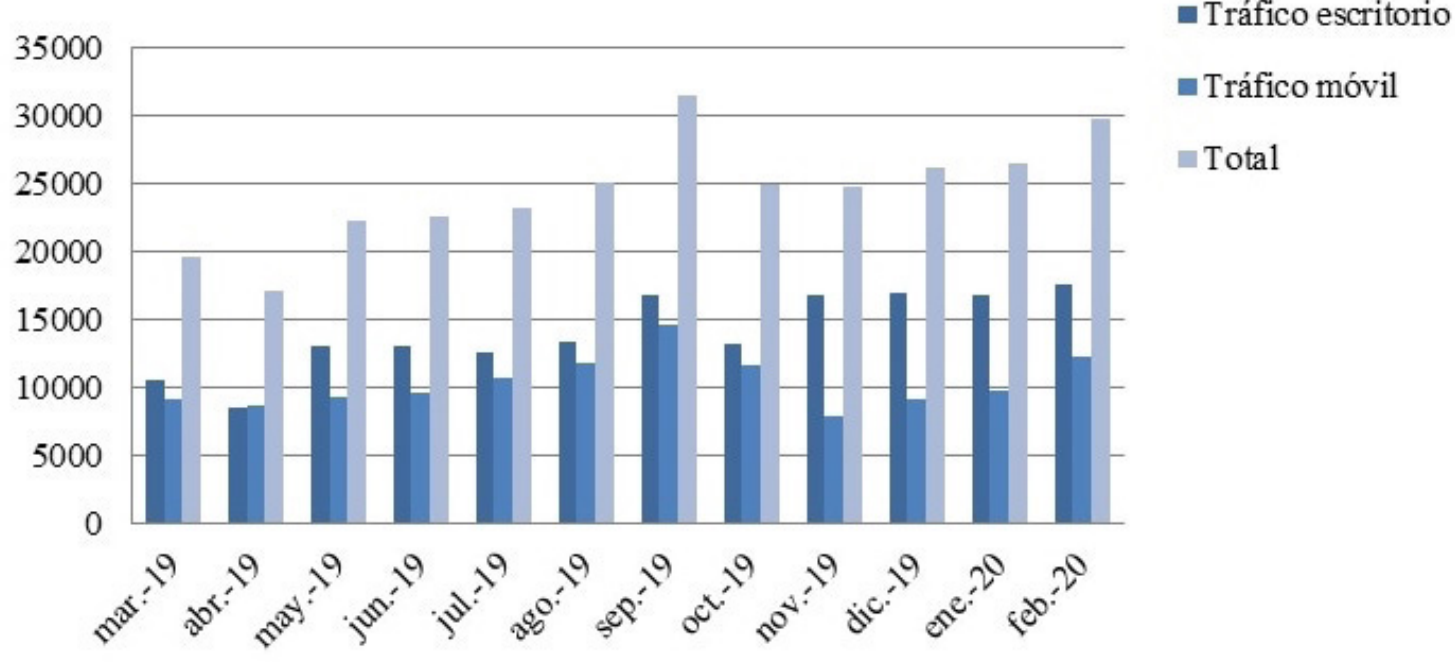


Tabla I: Número de anuncios por portal, palabra clave y dispositivo (escritorio o móvil)

\begin{tabular}{|c|c|c|c|c|c|c|c|}
\hline Dominio & ATFGE* & ATFGM* & ATFME* & ATFMM* & ATESE* & ATESM* & $\begin{array}{c}\text { Total } \\
\text { Anuncios }\end{array}$ \\
\hline gabinetedeestudios.com & 16 & 12 & 24 & 6 & 0 & 0 & 58 \\
\hline tesisymasters.es & 34 & 8 & 6 & 7 & 0 & 0 & 55 \\
\hline expertfg.com & 13 & 14 & 14 & 11 & 0 & 0 & 52 \\
\hline tfgmasters.es & 26 & 15 & 0 & 7 & 0 & 3 & 51 \\
\hline \begin{tabular}{|l|} 
tutfg.es \\
\end{tabular} & 15 & 14 & 12 & 9 & 1 & 0 & 51 \\
\hline apruebatodo.com & 28 & 3 & 12 & 6 & 0 & 0 & 49 \\
\hline apruebatfg.com & 20 & 5 & 15 & 8 & 0 & 0 & 48 \\
\hline tfgymasters.com & 36 & 9 & 0 & 0 & 0 & 0 & 45 \\
\hline gradostesisymasters.com & 25 & 0 & 8 & 11 & 0 & 0 & 44 \\
\hline tustrabajosatiempo.com & 20 & 9 & 8 & 5 & 0 & 0 & 42 \\
\hline tfgsherpa.com & 9 & 14 & 0 & 10 & 0 & 0 & 33 \\
\hline trabajosparauniversidad.com & 20 & 9 & 0 & 0 & 0 & 0 & 29 \\
\hline nohagasnada.com & 7 & 4 & 16 & 0 & 0 & 0 & 27 \\
\hline \begin{tabular}{|l|} 
tfgacademia.com \\
\end{tabular} & 19 & 4 & 0 & 0 & 0 & 0 & 23 \\
\hline academiauniversitaria.net & 8 & 11 & 0 & 4 & 0 & 0 & 23 \\
\hline apruebaya.es & 12 & 4 & 1 & 0 & 0 & 0 & 17 \\
\hline tumatriculadehonor.es & 4 & 4 & 6 & 3 & 0 & 0 & 17 \\
\hline asesoriatfgtfm.es & 0 & 4 & 0 & 11 & 0 & 0 & 15 \\
\hline gabinetedeestudios.es & 11 & 0 & 0 & 0 & 0 & 0 & 11 \\
\hline aprobarsinestudiar.com & 7 & 0 & 3 & 0 & 0 & 0 & 10 \\
\hline ayudauniversitaria.com & 2 & 4 & 0 & 1 & 0 & 0 & 7 \\
\hline lacasadeltfg.es & 0 & 3 & 0 & 4 & 0 & 0 & 7 \\
\hline universitariosenapuros.com & 0 & 1 & 0 & 6 & 0 & 0 & 7 \\
\hline apruebatodo.es & 0 & 4 & 0 & 2 & 0 & 0 & 6 \\
\hline ayudatfgtfm.es & 5 & 0 & 0 & 0 & 0 & 0 & 5 \\
\hline adeyderecho.com & 5 & & 0 & 0 & 0 & 0 & 5 \\
\hline redactorestfg.es & 4 & 0 & 0 & 0 & 0 & 0 & 4 \\
\hline avanzayformate.com & 4 & 0 & 0 & 0 & 0 & 0 & 4 \\
\hline consultoriadocente.com & 3 & 0 & 0 & 1 & 0 & 0 & 4 \\
\hline tfgexperts.es & 3 & 0 & 0 & 0 & 0 & 0 & 3 \\
\hline tustrabajosuniversitarios.com & 3 & 0 & 0 & 0 & 0 & 0 & 3 \\
\hline trabajosfindegrado.es & 0 & 1 & 1 & 0 & 0 & 0 & 2 \\
\hline aseguratugrado.com & 0 & 1 & 0 & 0 & 0 & 0 & 1 \\
\hline methodo.es & 0 & 0 & 0 & 0 & 1 & 0 & 1 \\
\hline asesoriatesis.com & 0 & 0 & 0 & 0 & 0 & 1 & 1 \\
\hline escribimos.com & 0 & 0 & 0 & 0 & 0 & 1 & 1 \\
\hline \begin{tabular}{|l|} 
Total \\
\end{tabular} & 359 & 157 & 126 & 112 & 2 & 5 & 761 \\
\hline
\end{tabular}

*Acrónimos: ATFGE Anuncios de TFG en Escritorio / ATFGM Anuncios de TFG en Móvil / ATFME Anuncios de TFM en Escritorio / ATFMM ATFME Anuncios de TFM en Móvil / ATESE Anuncios de Tesis en Escritorio / ATESM Anuncios de Tesis en Móvil

anuncios) y de Tesis (7 anuncios). Con estos datos, se evidencia que el "target group" más importante de estos portales es el alumnado que cursa el último año de estudios de Grado y tiene que realizar su TFG.

\subsection{Tráfico recibido por los portales de com- praventa de trabajos académicos}

Por lo que respecta al tráfico que reciben los portales analizados se observa una tendencia alcista durante los 12 meses analizados, con repuntes sig- nificativos en el mes de septiembre de 2019. La Figura 1 muestra los datos del tráfico recibido por los portales en su conjunto, diferenciando el recibido desde búsquedas en dispositivos de escritorio del de dispositivos móviles; puede observarse (ver también la Tabla II) que, en líneas generales, el tráfico de escritorio es algo superior (promedio mensual de 14.097 búsquedas de escritorio frente a 10.836 búsquedas móviles de media mensual, para un total de 24.933 búsquedas mensuales de promedio). En la Tabla II se ofrecen los datos mensuales y se ob- 
Tabla II: Tráfico mensual recibido por los portales a partir de búsquedas de escritorio y búsquedas móviles

\begin{tabular}{|l|c|c|c|}
\hline Mes & Tráfico búsqueda escritorio & Tráfico búsqueda móvil & Búsqueda total \\
\hline mar-19 & 10.525 & 9.128 & 19.653 \\
\hline abr-19 & 8.489 & 8.599 & 17.088 \\
\hline may-19 & 12.982 & 9.328 & 22.310 \\
\hline jun-19 & 13.013 & 9537 & 22.550 \\
\hline jul-19 & 12.578 & 10.667 & 23.245 \\
\hline ago-19 & 13.366 & 11.764 & 25.130 \\
\hline sep-19 & 16.844 & 14.664 & 31.508 \\
\hline oct-19 & 13.279 & 11.630 & 24.909 \\
\hline nov-19 & 16.823 & 7.899 & 24.722 \\
\hline dic-19 & 17.026 & 9.157 & 26.183 \\
\hline ene-20 & 16.735 & 9.740 & 26.475 \\
\hline feb-20 & 17.509 & 12.219 & 29.728 \\
\hline Total & 169.169 & 124.332 & 293.501 \\
\hline Promedio mensual & 14.097 & 10.361 & 24.458 \\
\hline Promedio mensual por portal & 391,5 & 287,8 & 679.3 \\
\hline
\end{tabular}

Figura 2: Tráfico orgánico y de pago recibido por los portales de compraventa de trabajos académicos.

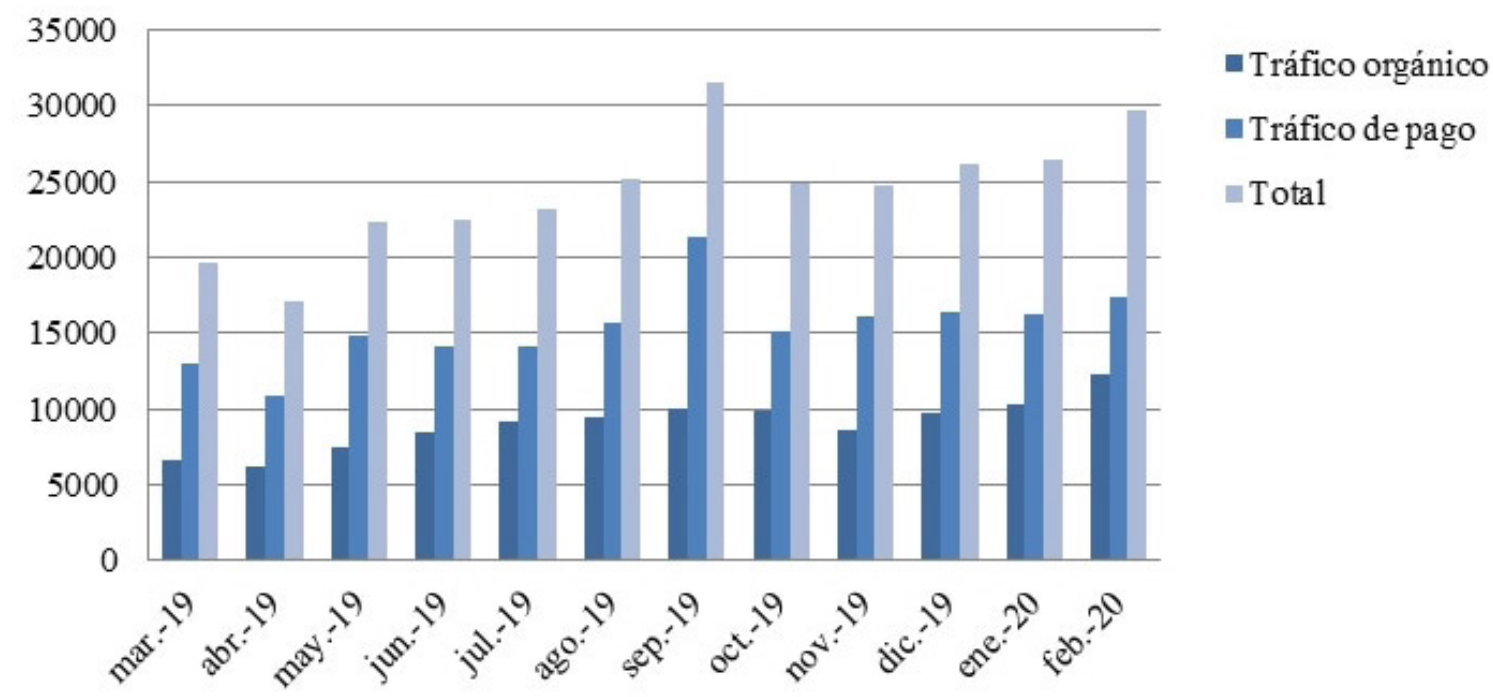

serva cómo el mínimo de tráfico a partir de búsquedas totales se produjo en abril de 2019 con 17.088 y el máximo en septiembre de 2019 con 31.508 .

Cabe destacar la notable diferencia que se observa entre el tráfico generado por búsquedas orgánicas o de pago (ver Figura 2 y Tabla III); siendo el segundo muy superior al primero.

El coste del tráfico de pago a partir de búsquedas en escritorio (no se dispone de datos del coste del tráfico móvil) alcanzó, para el total de portales analizados, un montante de 143.162€ (ver Figura
3). Ello supone una media mensual de $11.930 €$ y un promedio de $331 €$ por portal. En el Figura 3 se pueden observar los datos del coste de la publicidad online (calculada en base al tráfico de escritorio) soportados por los sitios web analizados; también en este caso se produce un considerable incremento en el mes de septiembre, aumento ya observado en el análisis del tráfico.

En cuanto a la relación entre el volumen de anuncios publicados y el tráfico de pago recibido, el resultado del análisis estadístico (Chi cuadrado 
Tabla III: Tráfico orgánico y de pago recibido por los portales

\begin{tabular}{|l|r|r|r|}
\hline Mes & \multicolumn{1}{|c|}{$\begin{array}{c}\text { Tráfico } \\
\text { orgánico }\end{array}$} & $\begin{array}{r}\text { Tráfico } \\
\text { de pago }\end{array}$ & Total \\
\hline mar-19 & 6.583 & 13.070 & 19.653 \\
\hline abr-19 & 6.151 & 10.937 & 17.088 \\
\hline may-19 & 7.438 & 14.872 & 22.310 \\
\hline jun-19 & 8.440 & 14.110 & 22.550 \\
\hline jul-19 & 9137 & 14.108 & 23.245 \\
\hline ago-19 & 9.437 & 15.693 & 25.130 \\
\hline sep-19 & 10.088 & 21.420 & 31.508 \\
\hline oct-19 & 9.852 & 15.057 & 24.909 \\
\hline nov-19 & 8.666 & 16.056 & 24.722 \\
\hline dic-19 & 9.810 & 16.373 & 26.183 \\
\hline ene-20 & 10.244 & 16.231 & 26.475 \\
\hline feb-20 & 12.354 & 17.374 & 29.728 \\
\hline Total & 108.200 & 185.301 & 293.501 \\
\hline Promedio mensual & 9.016 & 15.441 & 24.458 \\
\hline $\begin{array}{l}\text { Promedio mensual } \\
\text { por portal }\end{array}$ & 250,4 & 428,9 & 679.3 \\
\hline
\end{tabular}

de Pearson) arroja una relación positiva significativa: a mayor número de anuncios más tráfico de búsquedas de pago reciben los portales de compraventa de trabajos (ver Tabla IV).

En base a los datos de los 6 últimos meses se constata un incremento en el tiempo que pasan los visitantes en los portales analizados (tiempo total), no sólo en lo que se refiere a los datos netos

Tabla V: Duración media de las visitas y tasa de rebote de los portales analizados

\begin{tabular}{|l|c|c|}
\hline Mes & $\begin{array}{c}\text { Duración media de las } \\
\text { visitas (segundos) }\end{array}$ & $\begin{array}{c}\text { Tasa de } \\
\text { rebote }\end{array}$ \\
\hline sep-19 & 3.748 & $70,9 \%$ \\
\hline oct-19 & 3.810 & $70,3 \%$ \\
\hline nov-19 & 5.814 & $68,5 \%$ \\
\hline dic-19 & 4.941 & $75 \%$ \\
\hline ene-20 & 4.164 & $66,6 \%$ \\
\hline feb-20 & 5.147 & $68,5 \%$ \\
\hline Media total & 4.544 & $70 \%$ \\
\hline
\end{tabular}

Figura 3: Coste del tráfico de pago en Euros recibido por los portales de compraventa de trabajos académicos desde dispositivos de escritorio.

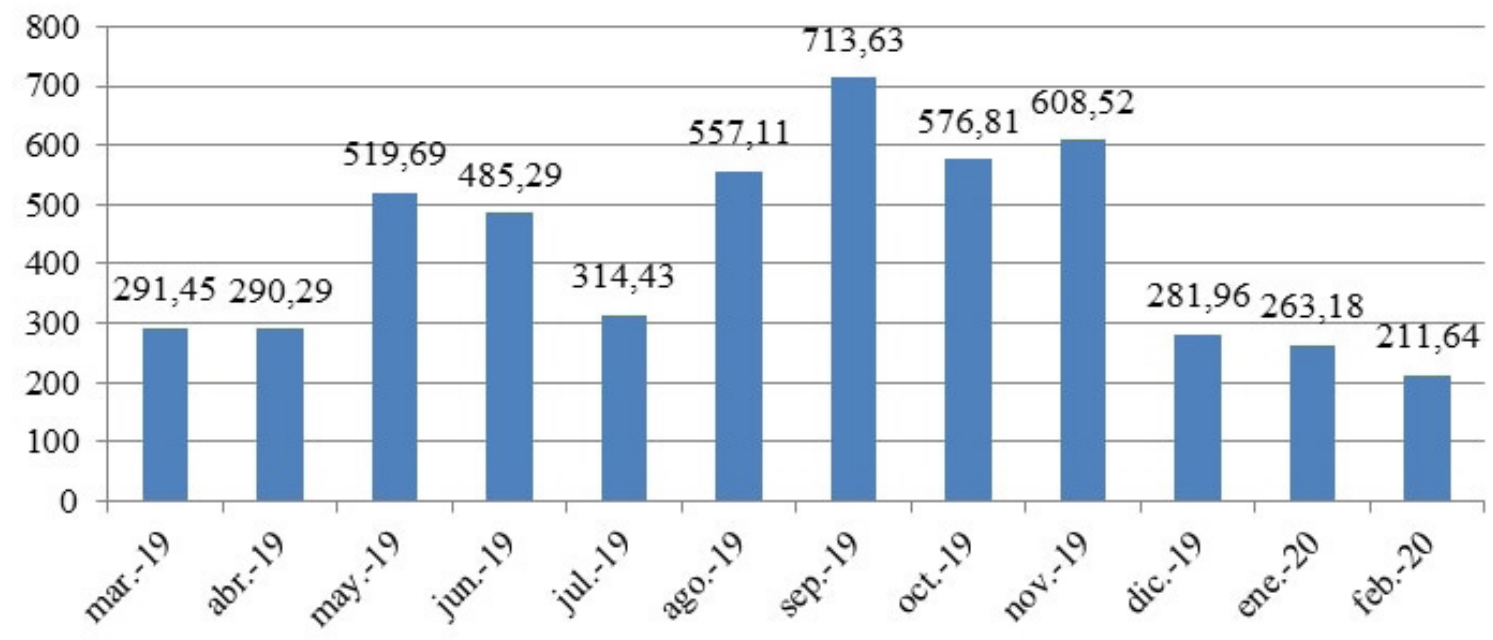

Tabla IV: Tráfico de pago recibido por los portales por anuncios publicados

\begin{tabular}{|c|c|c|c|c|c|c|}
\hline \multirow[b]{2}{*}{$\begin{array}{c}\text { Tráfico de búsqueda } \\
\text { de pago }\end{array}$} & \multicolumn{3}{|c|}{ Número de anuncios publicados en 12 meses } & \multirow[b]{2}{*}{$x^{2}$} & \multirow[b]{2}{*}{ g.l. } & \multirow[b]{2}{*}{$\boldsymbol{p}$} \\
\hline & $\begin{array}{l}\text { Horquilla alta } \\
\text { (portales con más } \\
\text { de } 40 \text { anuncios) }\end{array}$ & \begin{tabular}{|c|} 
Horquilla media \\
(portales con entre \\
10 y 39 anuncios)
\end{tabular} & $\begin{array}{c}\text { Horquilla baja } \\
\text { (portales con menos } \\
\text { de } 10 \text { anuncios) }\end{array}$ & & & \\
\hline Superior a $\mathbf{5 5 0 0}$ & $72,7 \%$ & $27,3 \%$ & $0 \%$ & \multirow{3}{*}{$20,441^{a}$} & \multirow{3}{*}{4} & \multirow{3}{*}{$.000^{*}$} \\
\hline Entre 1200 y 5499 & $16,7 \% \%$ & $33,3 \%$ & $50 \%$ & & & \\
\hline Inferior a 1200 & $0 \%$ & $23,1 \%$ & $76,9 \%$ & & & \\
\hline
\end{tabular}

$X^{2}=$ Chi-cuadrado de Pearson; $p=$ Significación; g.l. = Grados de libertad; * Significativa al nivel 0,01 (bilateral) 
Figura 4: Procedencia geográfica del tráfico recibido por los portales de compraventa de trabajos académicos.

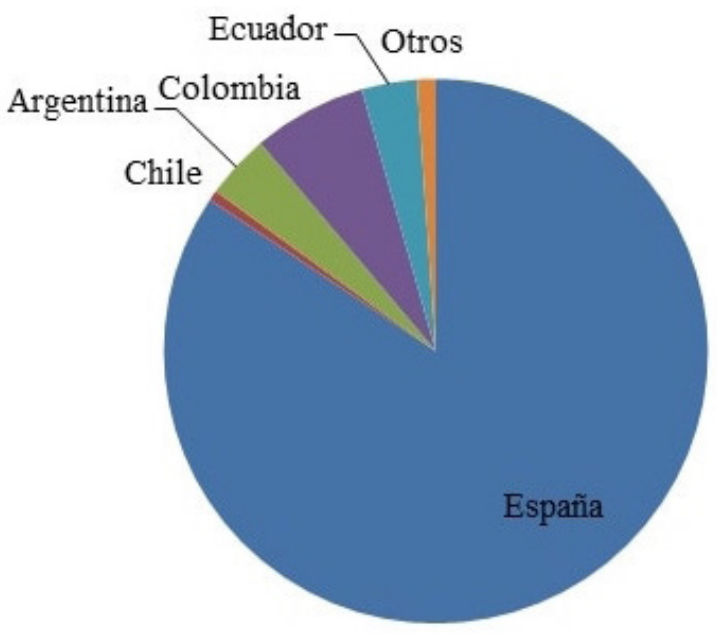

expresados en segundos sino también en los datos referidos a la tasa de rebote de las visitas (ver Tabla V). La duración media de visita a través de dispositivos de escritorio para cada portal se sitúa en los 126,2 segundos (unos dos minutos).

El tráfico que reciben los portales españoles de compraventa de trabajos académicos proviene mayoritariamente de España (84,4\%). El resto proviene de Colombia (7\%), Argentina (4\%), Ecuador $(3 \%)$, Chile $(1 \%)$, y el $1 \%$ restante de otros países (ver Figura 4).

\subsection{Análisis de las palabras clave y cadenas de palabras empleadas en los anuncios}

Del conjunto de palabras clave identificadas se seleccionaron aquellas que de manera abierta hacen referencia a la compraventa de trabajos académicos, obteniendo así un total de 39 ítems. Cada cadena de palabras se analizó mediante la aplicación "Análisis de palabra clave" obteniendo los resultados que se muestran en la Tabla VI. Obsérvese que el PPC de las palabras analizadas se sitúa, de media, en torno a $1 €$, promediando unos 29 anuncios por palabra clave durante el periodo analizado (12 meses).

El volumen de búsqueda orgánica generada por las palabras analizadas se sitúa entre las más de 300 búsquedas mensuales (es el caso de cadenas como "ayuda tfg", "comprar tfg" y "hacer tfg"; todas ellas centradas en los TFG), pasando por cadenas que acumulan algo más de 100 búsquedas mensuales (como "comprar tfm", "encargar tfg", "hacer $\mathrm{tfm}^{\prime \prime)}$, mientras que la mayoría de cadenas no llegan a las 100 búsquedas orgánicas mensuales.

\section{DISCUSIÓN Y CONCLUSIONES}

Los resultados obtenidos proporcionan evidencias sobre los procesos y modelos de publicidad online de las plataformas dedicadas a la comercialización de trabajos académicos 0 , si se quiere expresar con palabras más asépticas, a la escritura académica personalizada. Estos portales contribuyen de forma importante al problema del fraude en los procesos de evaluación en la enseñanza superior.

En este artículo se pone de relieve que los portales españoles de compraventa de trabajos académicos usan de manera extensiva el marketing digital; los casi 800 anuncios, recopilados en un periodo de 12 meses, que han sido la fuente de datos del trabajo y la inversión media que hacen estos portales de $331 €$ mensuales en publicitar sus servicios a través de Google Ads así lo atestiguan. También se constata que aquellos portales que más anuncios insertan reciben mayores cotas de tráfico; la publicidad, también en este sector de negocio, se erige como un eficiente reclamo para captar clientes (Kaktinš, 2018). Por otra parte, los anuncios demuestran su eficacia al asegurar un mayor movimiento; recordemos que el tráfico de pago es superior al orgánico, tanto mensualmente como en su globalidad. Además, dicha publicidad está claramente orientada al alumnado de los últimos cursos de Grado y en menor medida al de Máster y Doctorandos. No en vano el número de potenciales clientes a quien vender sus productos es mayor entre los estudiantes de Grado y la complejidad de los TFG es menor que la de los TFM o Tesis Doctorales y los procesos de evaluación son menos estrictos y la posibilidad de descubrir el fraude es menor.

En lo que respecta al tráfico que reciben estos portales se detecta un claro incremento a lo largo de los 12 meses analizados, siendo septiembre el mes en que se produce un mayor tráfico. Una explicación plausible puede ser la que relaciona las conductas deshonestas del alunado con la presión del último momento generada por la procrastinación (Ip y otros, 2016; Sureda y otros, 2015). Aquellos alumnos que deben entregar su TFG o TFM en la última convocatoria del curso y no lo tienen hecho sólo tienen, si quieren presentar su trabajo para ser evaluado, una posibilidad de salida (nada honrosa): comprar el trabajo y entregarlo como propio.

Por lo que respecta a las palabras clave, señalar que si el nombre comercial que usan muchas de estas empresas ya de por sí representa una clara incitación al fraude académico ("No hagas nada", "Aprobar sin estudiar", "Tus trabajos a tiempo"), qué decir de las cadenas de palabras usadas en sus 
Tabla VI: Palabras clave empleadas en los anuncios de los portales analizados

\begin{tabular}{|c|c|c|c|c|}
\hline Palabras clave o cadena & $\begin{array}{c}\text { Volumen de } \\
\text { búsqueda } \\
\text { orgánica mensual }\end{array}$ & PPC & $\begin{array}{c}\text { Número de } \\
\text { resultados en } \\
\text { búsqueda orgánica }\end{array}$ & $\begin{array}{c}\text { Número de } \\
\text { anuncios publicados } \\
\text { con esta palabra } \\
\text { clave en } 12 \text { meses } \\
\end{array}$ \\
\hline ayuda tfg & 390 & $1,16 €$ & 1.640 .000 & 62 \\
\hline ayuda trabajos universitarios & 90 & $0,56 €$ & 31.400 .000 & 18 \\
\hline comprar tfg & 390 & $1,26 €$ & 96 & 149 \\
\hline comprar tfm & 110 & $1,36 €$ & 373.000 & 66 \\
\hline corrección tfg & 40 & $0,85 €$ & 98 & 6 \\
\hline cuanto cuesta un tfg & 70 & $0,76 €$ & 90 & 13 \\
\hline elaboración de trabajos universitarios & 50 & $0,11 €$ & 63.100000 & 11 \\
\hline elaboración tesis & 40 & $0,47 €$ & 49.300 .000 & 25 \\
\hline empresas que te hacen el tfg & 70 & $1,41 €$ & 527.000 & 43 \\
\hline encargar tfg & 140 & $1,80 €$ & 87 & 53 \\
\hline encargar tfg derecho & 40 & $0,10 €$ & 60 & 4 \\
\hline encargar tfm & 90 & $1,89 €$ & 65 & 10 \\
\hline hacemos tesis & 50 & $0,44 €$ & 15.300 .000 & 18 \\
\hline hacemos tu tfg & 140 & $2,07 €$ & 121.000 & 64 \\
\hline hacemos tu trabajo universitario & 50 & $0,10 €$ & 33.600 .000 & 13 \\
\hline hacer el tfg en una semana & 50 & $0,79 €$ & 390.000 & 47 \\
\hline hacer tfg & 320 & $1,36 €$ & 1.700 .000 & 51 \\
\hline hacer tfg en un mes & 40 & $1,07 €$ & 3.410 .000 & 55 \\
\hline hacer tfm & 110 & $1 €$ & 776.000 & 12 \\
\hline hago tesis de grado & 40 & $0,28 €$ & 9.490 .000 & 33 \\
\hline $\begin{array}{l}\text { necesito que me hagan un trabajo de } \\
\text { la universidad }\end{array}$ & 90 & $2,30 €$ & 5100.000 & 21 \\
\hline personas que hacen tfg & 70 & $2,65 €$ & 2.050000 & 55 \\
\hline presupuesto tfg & 70 & $1,29 €$ & 310.000 & 17 \\
\hline quiero que me hagan el tfg & 90 & $1,80 €$ & 45 & 38 \\
\hline realización de tesis & 50 & $0,28 €$ & 56.800 .000 & 28 \\
\hline realización tfg & 40 & $1,40 €$ & 700.000 & 43 \\
\hline realizo proyectos fin de carrera & 90 & $\pm 0,10 €$ & 46.400 .000 & 8 \\
\hline tesis a domicilio & 30 & $\pm 0,10 €$ & 15.100 .000 & 5 \\
\hline tfg por encargo & 90 & $1,35 €$ & 89 & 47 \\
\hline tfg por encargo opiniones & 110 & $1,08 €$ & 63 & 3 \\
\hline tfm por encargo & 70 & $1,61 €$ & 88 & 11 \\
\hline tfm precio & 50 & $0,78 €$ & 560.000 & 2 \\
\hline trabajos universitarios online & 70 & $0,97 €$ & 13.100 .000 & 17 \\
\hline tu tfg a medida & 70 & $1,15 €$ & 88 & 33 \\
\hline realizo proyectos fin de carrera & 90 & $\pm 0,10 €$ & 46.400 .000 & 8 \\
\hline tesis por encargo & 50 & $0,59 €$ & 12.300 .000 & 15 \\
\hline se puede hacer un tfg en dos semanas & 50 & $1,09 €$ & 992.000 & 3 \\
\hline cuánto cuesta que te hagan un tfg & 50 & $1,02 €$ & 71 & 15 \\
\hline Total & 3550 & $38,20 €$ & 410.939 .940 & 1122 \\
\hline $\begin{array}{l}\text { Promedio por palabra clave } \\
\text { analizada }\end{array}$ & 91 & $0,97 €$ & 10.536 .000 & 28,7 \\
\hline
\end{tabular}


anuncios en Google Ads: "hacemos tfg", "hacemos tesis", "precio tfg", "hacemos tu trabajo universitario", etc. Los anuncios de estos portales, como se ha mostrado en otros trabajos centrados en analizar el contenido de los anuncios (Kaktinš, 2018) son una evidente instigación a que el alumnado contravenga los valores de integridad, ética, probidad y responsabilidad que deben ser los cimientos de su formación académica.

Poniendo la lupa sobre el alumnado cabe destacar que el análisis de las palabras clave sugiere que las búsquedas de ofertas de trabajos académicos en Internet es más que considerable. Una media mensual de 390 búsquedas orgánicas con el descriptor "comprar tfg" pone de manifiesto esta cuestión. El total de búsquedas orgánicas que se realizan usando descriptores que describen la compraventa de trabajos académicos son significativas: más de 3.500 veces se buscó este tipo de información en los 12 meses que contemplan los resultados de nuestro trabajo.

El trabajo que hemos llevado a cabo tiene un marcado carácter exploratorio, habiéndose centrado en el estudio de un objeto de análisis muy concreto y en un contexto muy determinado: el tráfico y la publicidad online de los portales de compraventa de trabajos académicos en España. Somos conscientes de que para poder reflejar de manera más certera la realidad en un escenario global interconectado como en el que vivimos sería conveniente adoptar perspectivas más globales y en un futuro planificamos replicar el estudio a una muestra superior de portales internacionales. Cabe señalar, sin embargo, que las diferencias culturales así como las diferentes tradiciones de los sistemas educativos nacionales pueden justificar análisis de ámbito geográfico limitado como el aquí presentado. En todo caso, nuestro trabajo sobre el tráfico e impacto de los procesos de marketing digital de los portales de escritura fantasma en España pueden constituir un primer paso para afrontar posteriores análisis de ámbito geográfico más amplio como hemos comentado.

Somos también conscientes de la limitación metodológica que supone trabajar con datos de fuentes secundarias, en nuestro caso de un programa de análisis de marketing digital. Consideramos, sin embargo, que estos datos son altamente fiables y por ello susceptibles de ser usados en estudios empíricos, como recientemente se ha hecho en otros trabajos (Lopezosa y otros, 2019a; Lopezosa y otros, 2019b; Lopezosa y otros, 2020; Vyas, 2019).

Otra dificultad con la que nos enfrentamos fue la falta de datos en SEMrush del tráfico de pago originado a través de dispositivos móviles para el total del periodo analizado; dificultad que sorteamos recurriendo a la media de los siete meses de los que sí se disponía de datos. Reconocemos que este cálculo puede haber originado alteraciones en el cómputo total y en los resultados, aunque consideramos que se trata de variaciones leves que no distorsionan los datos y evidencias obtenidas.

Sobre esta cuestión entendemos que herramientas como SEMrush pueden ser muy útiles en estudios del área de la información y la documentación, entre otros. Un buen ejemplo del potencial de este tipo de herramientas en estudios empíricos queda claramente reflejado en nuestro aporte: no se dispone de evidencias acerca de cuántos trabajos académicos se venden en España y las estimaciones a nivel internacional son también muy confusas ya que difícilmente las empresas dedicadas a este "negocio" aportan datos acerca del mismo (Newton, 2018) e igualmente es poco probable que los clientes de estos servicios lo comuniquen si se les pregunta al respecto (Lancaster y Clarke, 2016). Nuestro estudio no resuelve la cuestión, (no podemos precisar el número de operaciones de compraventa), pero sí permite precisar el volumen de tráfico de los portales, establecer un coste mensual por publicidad y conocer qué y cuántas veces se buscan trabajos académicos en venta a través de Internet. Creemos que, sobre todo, esta última posibilidad es altamente interesante y aplicable a otros campos de estudio sobre búsquedas y consumo de información en la Red. Además, consideramos que, esta posibilidad (conocer qué se busca y con qué frecuencia), puede servir también para comparar perfiles de búsqueda de información en diferentes países y en diferentes momentos para analizar su evolución, por ejemplo.

En cuanto a las conclusiones, señalar, en primer lugar, la relevancia de haber identificado los dominios de un total de 36 portales españoles dedicados a la compraventa de trabajos académicos que usan los servicios de publicidad de Google. Esta identificación constituye también una denuncia que debería ser escuchada por el propio buscador. Al publicar anuncios de webs de escritura fantasma, Google infringe abiertamente su propia política de publicidad; una política que en lo relativo a "Posibilitar el comportamiento fraudulento" señala:

Valoramos la honestidad y la justicia, de manera que no permitimos que se promocionen productos o servicios diseñados para admitir un comportamiento deshonesto. Ejemplos de productos o servicios que posibilitan un comportamiento fraudulento: software o instrucciones de piratería; servicios diseñados para aumentar artificialmente el tráfico del anuncio o del sitio 
web; documentos falsos; servicios de fraudes académicos (Google Ads, 2020).

Consideramos que el buscador debería tomar buena nota de los resultados de nuestro estudio y ajustarse a su política de publicidad y no anunciar unos servicios que claramente contravienen sus propias normas. Este ejercicio de coherencia supondría para Google renunciar a unos ingresos que como hemos evidenciado en nuestro trabajo alcanzan algo más de $140.000 €$ anuales (sólo contabilizando los datos del tráfico de pago de escritorio) para el contexto español.

Por otra parte, las administraciones competentes deberían encarar el desafío que plantea la publicidad de los servicios de escritura académica y apostar por su prohibición, como se ha hecho en Irlanda, Nueva Zelanda y Australia (Bretag, 2019). Cabe remarcar, sin embargo, que incluso en estos países con legislaciones coercitivas los enjuiciamientos son poco frecuentes, en gran parte debido a lagunas de las disposiciones legales. Probablemente, el caso más conocido se dio en Nueva Zelanda, que en 2018 procesó con éxito el servicio comercial Assignments $4 \mathrm{U}$, que acabó pagando 1.3 millones de dólares estadounidenses en un acuerdo extrajudicial y el posterior cierre del portal (Owen, 2018).

Hay que tener en cuenta que la prohibición de este tipo de portales por parte de un Estado no necesariamente supondría su desaparición; lo más probable es que, como se ha producido con el negocio de la piratería digital (Juez, 2018), cambien el dominio y el emplazamiento de la empresa, radicándose en países con legislaciones más laxas.

No conocemos trabajos que hayan analizado la situación jurídica de los portales de escritura fantasma en España. Pero sí que sabemos de un conjunto de denuncias de clientes de los servicios de escritura fantasma dirigidas a la Organización de Consumidores y Usuarios (OCU) al considerarse víctimas de fraude e incumplimiento de contrato (pueden acceder a algunas de estas denuncias en los siguientes enlaces: https://bit.ly/3aoq7tm, https://bit.ly/2xozYAU, https://bit.ly/2wD6peT, https://bit.ly/2xm5DTz).

Los sitios web de compraventa de trabajos incitan al alumnado a desarrollar comportamientos que si bien no podemos calificar de ilegales por la falta de una legislación clara, suponen una falta grave de deshonestidad académica. Para esta incitación usan una lógica marcada por dos momentos bien analizados en el campo del marketing y la persuasión (Moran y Hunt, 2014). En una primera fase se presentan como reconocedores de los proble- mas de sus potenciales clientes; para ello ponen el énfasis en el estrés que soporta el alumnado ante las evaluaciones, definen como problema los trabajos que deben realizar, incluso como una pérdida de tiempo o una fuente de aburrimiento (Rowland y otros, 2018). En la segunda fase, se presentan como depositarios de la solución al problema, con apelaciones del tipo "nosotros trabajamos por ti, despreocúpate que nosotros nos ocupamos, somos profesionales y aportamos garantías de calidad, etc.". Esta forma de presentación de las plataformas proporciona al alumnado el argumentario necesario para que en la balanza valorativa de las implicaciones éticas de comprar un trabajo de evaluación pueda pesar más la "racionalización egoísta" que los principios éticos o morales (Murdoch y otros, 2016).

En este sentido, y como recomendaciones finales, creemos necesario, en primer lugar, que se visibilice el problema en nuestro país y a ello entendemos que ayuda nuestro estudio. En segundo lugar, insistimos en la necesidad de poner coto a la publicidad de los servicios de compraventa de trabajos académicos y a ello seguramente podría dedicar esfuerzos y ejercer presión ante la Administración la Conferencia de Rectores de las Universidades Españolas (CRUE). Al mismo tiempo, creemos necesaria la introducción en los reglamentos académicos de las universidades de normativas claras acerca de la gravedad que supone la compraventa de trabajos y la penalización a la que se pueden enfrentar los alumnos en caso de infringir la normativa; un trabajo reciente de Sureda, y otros (2007) pone muy en entredicho la funcionalidad y actualidad de los reglamentos académicos de las universidades españolas en lo concerniente a las conductas deshonestas por parte del alumnado. Por último, conviene reflexionar acerca de los procesos de evaluación y tutorización de los trabajos de evaluación; la adaptación de los planes de estudio al Plan Bolonia ha provocado un cambio metodológico marcado sobre todo por la evaluación continua y el trabajo autónomo del alumnado para el que muchos estudios demuestran que los alumnos no disponen de las competencias informacionales adecuadas para llevarlos a cabo eficazmente (Pinto y Sales, 2015; Rodrigues, 2014). Sobre este último punto, creemos relevante poner el acento en la conveniencia de no focalizar el estudio del tema solo en la oferta sino también sobre las características de la demanda y las variables o razones que la sustentan: ¿por qué se producen casi 400 búsquedas orgánicas mensuales con el descriptor "comprar TFG" en España? ¿qué lleva a un/a alumno/a a buscar en la Red trabajos que pueda comprar para obtener una titulación? 
En resumen, y refiriéndonos a la hipótesis que ha orientado este trabajo con los datos obtenidos se confirma que en los últimos años las plataformas españolas de compraventa de trabajos académico han conocido un importante aumento de tráfico y en este incremento los anuncios han tenido y tienen una alta incidencia dado que las búsquedas de pago son muy superiores a las búsquedas orgánicas. Los portales analizados invierten una considerable cantidad de recursos económicos en la publicidad en buscadores y las palabras clave utilizadas por los anuncios de estos portales constituyen una incitación al fraude académico. Remarcar, finalmente, que la solución al problema global de las fábricas de trabajos académicos demanda un enfoque holístico y multidisciplinar (Newton y Lang, 2016; Amigud y Dawson, 2020); un enfoque que implica al profesorado, al alumnado, a las autoridades académicas y a los legisladores.

\section{AGRADECIMIENTOS}

Este trabajo (Referencia: RTI2018-098314B-I00) ha sido financiado por el Ministerio de Ciencia e Innovación (MCI), la Agencia Estatal de Investigación (AEI) y el Fondo Europeo de Desarrollo Regional (FEDER).

\section{ACKNOWLEDGEMENTS}

This research (Reference: RTI2018-098314B-IO0) has been funded and supported by the Spanish Ministry of Science and Innovation (MCI), the National Research Agency (AEI) and the European Regional Development Fund (ERDF).

\section{NOTAS}

${ }^{1}$ Del tráfico móvil de pago sólo se dispuso, a la hora de realizar el estudio, de los datos de SEMrush de los últimos 7 meses (Agosto 2019 a Febrero 2020), por lo que para poder calcular el dato de los 5 meses restantes se aplicó la media de tráfico de dichos 7 meses y se usó como cálculo de tráfico de los meses de Marzo de 2019 a Julio de 2019.

\section{REFERENCIAS}

Amigud, A. (2020). Cheaters on Twitter: an analysis of engagement approaches of contract cheating services. Studies in Higher Education, 45(3), 692-705. https:// doi.org/10.1080/03075079.2018.1564258

Amigud, A.; Dawson, P. (2020). The law and the outlaw: is legal prohibition a viable solution to the contract cheating problem? Assessment \& Evaluation in Higher Education, 45(1), 98-108. https://doi.org/10.1080/02 602938.2019.1612851

Aregay, T. (2014). Ranking Factors for Web Search: Case Study in the Netherlands. [Tesis de Máster]. Holanda: University of Twente. https://bit.ly/2NouiMc [Fecha de consulta: 22/06/2020].
Aswani, R.; Kar, A.K.; Ilavarasana, P.V.; Dwivedi, Y.K. (2018). Search engine marketing is not all gold: Insights from Twitter and SEOClerks. International Journal of Information Management, 38(1), 107-116. https:// doi.org/10.1016/j.ijinfomgt.2017.07.005

Awdry, R.; Newton, P.M. (2019). Staff views on commercial contract cheating in higher education: a survey study in Australia and the UK. Higher Education. 78, 593-610 https://doi.org/10.1007/s10734-01900360-0

Baye, M.R.; De los Santos, B.; Wildenbeest, M.R. (2016). Search engine optimization: What drives organic traffic to retail sites? Journal of Economics \& Management Strategy, 25(1), 6-31. https://doi.org/10.1111/ jems.12141

Boughton, S.B. (2005). Search engine marketing. Perspectives in Business, 2(1), 29-33. https://bit.ly/3atnUx3 [Fecha de consulta: 30/03/2020].

Bretag, T. (2019). Contract cheating will erode trust in science. Nature, 574(7780), 599. https://doi. org/10.1038/d41586-019-03265-1

Bretag, T.; Harper, R.; Burton, M.; Ellis, C.; Newton, P.; Rozenberg, P.; van Haeringen, K. (2018). Contract cheating: a survey of Australian university students. Studies in Higher Education, 44(11), 1837-1856. https://doi.org/10.1080/03075079.2018.1462788

Bretag, T.; Harper, R.; Rundle, K.; Newton, P.M.; Ellis, C.; van Haeringen, K. (2020). Contract cheating in Australian higher education: a comparison of non-university higher education providers and universities. Assessment \& Evaluation in Higher Education, 45(1), 125-139. https://doi.org/10.1080/02602938.2019.16 14146

Chaffey, D. (2020). Search engine statistics, 2020. Disponible en https://www.smartinsights.com/search-engine-marketing/search-engine-statistics/ [Fecha de consulta: 08/05/2021]

Clare, J.; Walker, S.; Hobson, J. (2017). Can we detect contract cheating using existing assessment data? Applying crime prevention theory to an academic integrity issue. International Journal for Educational Integrity, 13(1). https://doi.org/10.1007/s40979-0170015-4

Comas, R. (2009). El ciberplagio y otras formas de deshonestidad académica entre el alumnado universitario. [Tesis doctoral]. España: Universidad de las Islas Baleares.

Curtis, G.J.; Clare, J. (2017). How Prevalent is Contract Cheating and to What Extent are Students Repeat Offenders? Journal of Academic Ethics, 15(2), 115124. https://doi.org/10.1007/s10805-017-9278-x

Dawson, P.; Sutherland-Smith, W. (2018). Can markers detect contract cheating? Results from a pilot study. Assessment and Evaluation in Higher Education, 43(2), 286-293. https://doi.org/10.1080/02602938.2 017.1336746

Dawson, P.; Sutherland-Smith, W. (2019). Can training improve marker accuracy at detecting contract cheating? A multi-disciplinary pre-post study. Assessment and Evaluation in Higher Education, 44(5), 715-725. https://doi.org/10.1080/0260293 8.2018 .1531109 
Devlin, M.: Gray, K. (2007). In Their Own Words: A Qualitative Study of the Reasons Australian University Students Plagiarize. Higher Education Research and Development, 26(2), 181-198. https://doi. org/10.1080/07294360701310805

Draper, M.J.; Newton, P.M. (2017). A legal approach to tackling contract cheating? International Journal for Educational Integrity, 13(1). https://doi.org/10.1007/ s40979-017-0022-5

Draper, M.J.; Ibezim, V.; Newton, P.M. (2017). Are Essay Mills committing fraud? An analysis of their behaviours vs the 2006 Fraud Act (UK). International Journal for Educational Integrity, 13(1). https://doi.org/10.1007/ s40979-017-0014-5

Dreller, J. (2010). A brief history of paid search advertising. Disponible en https://bit.ly/3dEdxbr [Fecha de consulta: 30/03/2020]

Drivas, I.C.; Sarlis, A.S.; Sakas, D.P.; Varveris, A. (2017). Stuffing Keyword Regulation in Search Engine Optimization for Scientific Marketing Conferences. En Kavoura A.: Sakas D.; Tomaras P. (eds) Strategic Innovative Marketing (pp. 117-123). Springer. https://doi. org/10.1007/978-3-319-56288-9_17

Ellis, C.; Zucker, I.M.; Randall, D. (2018). The infernal business of contract cheating: understanding the business processes and models of academic custom writing sites. International Journal for Educational Integrity, 14(1). https://doi.org/10.1007/s40979-017-0024-3

Foltýnek, T.; Králíková, V. (2018). Analysis of the contract cheating market in Czechia. International Journal for Educational Integrity, 14(1). https://doi.org/10.1007/ s40979-018-0027-8

Gavilán, D.; Martínez-Navarro, G.; Fernández-Lores, S. (2018). Influencia social en las comunidades de cine: filmaffinity como caso de estudio. Estudios sobre el Mensaje Periodistico, 24(1), 551-565. https://doi. org/10.5209/ESMP.59966

Goldfarb, A.; Tucker, C. (2011). Online display advertising: Targeting and obtrusiveness. Marketing Science, 30(3), 389-404. https://doi.org/10.1287/ mksc. 1100.0583

González-Soltero, R.; Blanco, M.J.; Biscaia, J.M.; Mohedano, R.B.; Grille-Mariscal, M.; Blanco, M.A. (2015). Análisis del contenido, posicionamiento y calidad de páginas web en español relacionadas con la nutrición y los trastornos de la conducta alimentaria. Nutrición Hospitalaria, 31(3), 1394-1402. http://dx.doi. org/10.3305/nh.2015.31.3.8122

Google Ads. (2020). Políticas de Google Ads. Disponible en https://bit.ly/3ass5cg [Fecha de consulta: 30/03/2020]

Gullifer, J. (2010). Exploring university students' perceptions of plagiarism: A focus group study. Studies in Higher Education, 35(4), 463-481. https://doi. org/10.1080/03075070903096508

Ip, E.; Nguyen, K.; Shah, B.; Doroudgar, S.; Bidwal, M. (2016). Motivations and predictors of cheating in pharmacy school. American Journal of Pharmaceutical Education, 80(8), 133. https://doi.org/10.5688/ ajpe808133 https://doi.org/10.5688/ajpe808133

Jafarzadeh, H.; Abedin, B.; Aurum, A.; D'Ambra, J. (2019). Search Engine Advertising Perceived Effec- tiveness: A Resource-Based Approach on the Role of Advertisers' Competencies. Journal of Organizational and End User Computing, 31(4), 46-73. https://doi. org/10.4018/JOEUC.2019100103

Jansen, B.J.: Spink, A. (2007). Sponsored search: is money a motivator for providing relevant results?. Computer, 40(8), 52-57. https://doi.org/10.1109/MC.2007.290

Juez, I. (2018). ¿Cómo logran las webs de descargas ilegales burlar a la Policía?, El Correo. Disponible en https://bit.ly/39qIDBf [Fecha de consulta: 30/03/2020]

Kaktinš, L. (2018). Contract cheating advertisements: what they tell us about international students' attitudes to academic integrity. Ethics and Education, 13(2), 268-284. https://doi.org/10.1080/17449642.2017.1412178

Kireyev, P.; Pauwels, K.; Gupta, S. (2016). Do display ads influence search? Attribution and dynamics in online advertising. International Journal of Research in Marketing, 33(3), 475-490. https://doi.org/10.1016/j. ijresmar.2015.09.007

Kritzinger, W.T.; Weideman, M. (2017). Parallel search engine optimisation and pay-per-click campaigns: A comparison of cost per acquisition. South African Journal of Information Management, 19(1). https://doi. org/10.4102/sajim.v19i1.820

Lancaster, T.; Clarke, R. (2016). Contract Cheating: The Outsourcing of Assessed Student Work. En: Bretag T. (eds) Handbook of Academic Integrity. Singapore: Springer. 639654. https://doi.org/10.1007/978-981-287-098-8_17

Lopezosa, C.; Codina, L.; Rovira, C. (2019a). Visibilidad web de portales de televisión y radio en España: ¿qué medios llevan a cabo un mejor posicionamiento en buscadores? Barcelona: Universitat Pompeu Fabra, Departamento de Comunicación; Ediciones Profesionales de la Información. Disponible en https://bit.ly/2UH3fig [Fecha de consulta: 30/03/2020]

Lopezosa, C.; Codina, L.; López-García, G.; Corbella-Cordomi, J.M. (2020). Mapa de visibilidad y posicionamiento en buscadores de los principales grupos mediáticos españoles. El profesional de la información (EPI), 29(2). https://doi.org/10.3145/epi.2020.mar.03

Lopezosa, C.: Orduna-Malea, E.; Pérez-Montoro, M. (2019b). Making Video News Visible: Identifying the Optimization Strategies of the Cybermedia on YouTube Using Web Metrics. Journalism Practice. https://doi.or $\mathrm{g} / 10.1080 / 17512786.2019 .1628657$

Marvin, G. (2017). Report: Google earns $78 \%$ of $\$ 36.7 B$ US search ad revenues, soon to be $80 \%$. Disponible en https://bit.ly/2wMGGkf [Fecha de consulta: 30/03/2020]

Moran, M.; Hunt, B. (2014). Search engine marketing, Inc.: Driving search traffic to your company's website. New Jersey: IBM Press.

Murdock, B.; Stephens, J.M.; Grotewiel, M. (2016). Student Dishonesty in the Face of Assessment. En Brown G. y Harris L. (eds) Handbook of human and social conditions in assessment. Londres: Routledge. 186-203

Nabout, N.A.; Lilienthal, M.; Skiera, B. (2014). Empirical generalizations in search engine advertising. Journal of Retailing, 90(2), 206-216. https://doi.org/10.1016/j. jretai.2014.03.002

Newton, P.M. (2018). How common is commercial contract cheating in higher education and is it increasing? 
A systematic review. Frontiers in Education, 3. https:// doi.org/10.3389/feduc. 2018.00067

Newton, P. M.; Lang, C. (2016). Custom essay writers, freelancers, and other paid third parties. En: Bretag T. (eds) Handbook of Academic Integrity. Singapore: Springer. 249-271. https://doi.org/10.1007/978-981287-098-8 17.

Owen, C. (2018). Multi-million dollar university 'assignment cheating' business in court. Disponible en https://bit.ly/3bG2ddj [Fecha de consulta: 30/03/2020]

Pinto, M.; Sales, D. (2015). Uncovering information literacy's disciplinary differences through students' attitudes: An empirical study. Journal of Librarianship and Information Science, 47(3), 204-215. https://doi. org/10.1177/0961000614532675

Rodrigues, R. (2014). Búsqueda, selección y gestión de información académica de los nativos digitales: pocas sorpresas y grandes retos educativos. Digital Education Review, 26, 39-60. https://revistes.ub.edu/index. php/der/article/download/11580/14440 [Fecha de consulta: 07/05/2021]

Rowland, S.; Slade, C.; Wong, K.S.; Whiting, B. (2018). 'Just turn to us': the persuasive features of contract cheating websites. Assessment \& Evaluation in Higher Education, 43(4), 652-665. https://doi.org/10.1080/0 2602938.2017.1391948

Sen, R. (2005). Optimal search engine marketing strategy. International Journal of Electronic Commerce, 10(1), 9-25. https://doi.org/10.1080/10864415.200 5.11043964
Sivasubramaniam, S.; Kostelidou, K.; Ramachandran, S. (2016). A close encounter with ghost-writers: an initial exploration study on background, strategies and attitudes of independent essay providers. International Journal for Educational Integrity, 12(1). https://doi.org/10.1007/s40979-016-0007-9

Sureda, J.; Comas, R.; Mut, T. (2007). Las "fábricas" de trabajos académicos: una incitación al fraude en las aulas. Informes de Investigación del Grupo "Educación y Ciudadanía". Disponible en https://docplayer. es/storage/38/17977256/1620420577/a XU00hnFz7jHI9u-JtFag/17977256.pdf [Fecha de consulta: 07/05/2021]

Taylor, Z.W. (2020), Optimizing Away Summer Melt: How Search Engine Optimization May Reduce Summer Melt at Highly Ranked US Institutions of Higher Education. Strategic Enrollment Management Quarterly (Abril 2020, pre-print). Disponible en https:// bit.ly/2QV2mSc [Fecha de consulta: 30/03/2020]

Vyas, C. (2019). Evaluating state tourism websites using Search Engine Optimization tools. Tourism Management, 73, 64-70. https://doi.org/10.1016/j. tourman.2019.01.019

Wallace, M.J.; Newton, P.M. (2014). Turnaround time and market capacity in contract cheating. Educational Studies, 40(2), 233-236. https://doi.org/10.108 $0 / 03055698.2014 .889597$

Zhang, S.; Cabage, N. (2017). Search engine optimization: Comparison of link building and social sharing. Journal of Computer Information Systems, 57(2), 148-159. https://doi.org/10.1080/08874417.2016.1183447 